\title{
Recomendaciones para el reinicio de cirugía ortopédica electiva post-COVID-19 en México
}

\author{
Guidelines for restarting elective orthopaedic surgery post COVID-19 in Mexico \\ Sommer-Larraza G,* Mallen-Trejo A,* Tomás-Reyna C, ${ }^{\ddagger}$ Rodríguez-Reséndiz F* \\ Centro Médico ABC.
}

\begin{abstract}
RESUMEN. Justificación: Proporcionar recomendaciones actuales que nos permitan retomar la cirugía ortopédica electiva posterior a la pandemia COVID-19 en condiciones adecuadas de seguridad para el personal de salud, pacientes y familiares para el tratamiento perioperatorio de acuerdo a la situación en nuestro país. Métodos: Revisión bibliográfica de literatura actual mundial relevante. Resultados: Una guía de recomendaciones con la adecuada definición de procedimientos ortopédicos electivos, la correcta estratificación de riesgo de contagio por COVID-19, las consideraciones especiales en selección de pacientes según su grupo de riesgo, las adecuaciones hospitalarias a implementar y los cuidados anestésicos, intraoperatorios y postoperatorios especiales ante el reinicio de cirugía electiva posterior a la pandemia COVID-19. Conclusiones: El reinicio seguro de cirugía ortopédica electiva posterior a la pandemia COVID-19 en México es posible tomando en cuenta las recomendaciones especiales preoperatorias, intraoperatorias y postoperatorias.
\end{abstract}

Palabras clave: COVID, SARS-CoV-2, México, cirugía electiva, cirugía ortopédica.

\section{Introducción}

En Diciembre de 2019 surgió en Wuhan, China un brote de neumonía que identificó el 31 de Diciembre de

\section{Nivel de evidencia: $\mathrm{V}$}

* Cirujano Ortopedista, Centro Médico ABC.

* Anestesióloga, Centro Médico ABC.

Dirección para correspondencia:

Gerd Sommer-Larraza

Emiliano Zapata 13c int 102, San Lucas Tepetlacalco, Tlanepantla de Baz CP 54055, Estado de México.

E-mail: info@ortopediasommer.com

Citar como: Sommer-Larraza G, Mallen-Trejo A, TomásReyna C, Rodríguez-Reséndiz F. Recomendaciones para el reinicio de cirugía ortopédica electiva post-COVID-19 en México. Acta Ortop Mex. 2020; 34(3): 176-182. https:// dx.doi.org/10.35366/97070
ABSTRACT. Background: Elaborate an updated guideline of recommendations for the safe return to elective orthopedic surgery post COVID-19 pandemic. Methods: Bibliographic review of relevant global literature. Results: Recommendation guidelines with appropriate definitions of orthopedic elective surgery, correct contagion risk stratification for COVID-19, considerations for specific risk groups, hospital adaptations and anesthetic, intraoperative and postoperative special care for a safe restart of orthopedic elective surgery post COVID-19 pandemic. Conclusions: The safe restart of orthopedic elective surgery is possible as long as we take into consideration the appropriate recommendations, which we have summarized in this review.

Keywords: COVID, SARS-CoV-2, Mexico, elective surgery, orthopedic surgery.

2019 una nueva cepa de coronavirus denominado 2019nCoV SARS-CoV-2 por la OMS (Organización Mundial de la Salud). El virus se esparció rápidamente a nivel global y fue declarado como pandemia COVID-19 el 11 de Marzo de 2020. En nuestro país, ante el inminente riesgo de numerosas pérdidas humanas se diseñaron estrategias para aplanar la curva de contagio y así evitar la saturación de servicios hospitalarios, entre ellas, se suspendieron las cirugías electivas, las cuales con el tiempo tendrán que ser retomadas. Sin embargo, tenemos que ser especialmente cuidadosos para evitar que el reinicio de las cirugías programadas fomente un segundo pico en la curva de contagio. No está bien definido el tiempo adecuado para el reinicio de las cirugías programadas, pero el consenso general es esperar dos semanas posteriores al declive de incidencia de contagio por COVID-19. Realizamos una búsqueda de la literatura, encontrando las siguientes recomendaciones para retomar las actividades previas de la manera más segura posible. 


\section{Definiciones}

La Academia Americana de Cirujanos Ortopedistas AAOS define cuatro grados de urgencia para cirugía ortopédica (Tabla 1). ${ }^{1}$

Las categorías A y B necesitan manejo oportuno a pesar de la situación de emergencia sanitaria y no forman parte de esta revisión. Nos enfocaremos en las categorías C) y D), cirugías diferibles sin comprometer la salud y/o grado funcional del paciente.

\section{Condiciones iniciales}

Antes de tomar acción propia, es de vital importancia que la parte médica trabaje en conjunto con la parte administrativa para que las instalaciones sanitarias estén preparadas para el reinicio de la cirugía electiva. Lo ideal es que el hospital designe áreas libres de COVID-19, en las que se incluyan zonas para el ingreso, hospitalización, transporte adecuado del paciente a quirófano y posteriormente su habitación y que garantice los recursos necesarios en caso de cualquier complicación o emergencia con disponibilidad de camas en terapia intensiva y ventiladores. El equipo quirúrgico, de enfermería y transporte de pacientes debe valorarse y de ser posible, someterse a pruebas de reacción en cadena de la polimerasa (PCR por su siglas en inglés) para COVID-19 para mantener estas áreas lo más seguras posible.

\section{Preoperatorio}

Entablar una relación con la parte administrativa de los hospitales para que los trámites de ingreso y cuestiones administrativas se realicen directamente en la habitación donde estará el paciente y así evitar mayores contactos en zonas comunes; el paciente deberá usar incondicionalmente cubrebocas durante su estancia hospitalaria.

\section{Selección de pacientes}

La selección de pacientes es crucial para mantener la seguridad durante el reinicio de procedimientos electivos.

Inicialmente se debe estratificar el riesgo de exposición del paciente, de acuerdo a Fineberg ${ }^{2}$ puede definirse en cinco grupos resumidos en la Tabla 2.
Se debe integrar a la valoración preoperatoria tradicional una anamnesis dirigida a los principales síntomas por infección por COVID-19. Los signos y síntomas más comunes con sus respectivos porcentajes reportados son los siguientes: fiebre (77-100\%), tos (48-75\%), fatiga (39-70\%), disnea (28-50\%), mialgias/artralgias (17-32\%), cefalea (5-30\%), hiposmia y/o hipogeusia (30\%) y síntomas gastrointestinales (diarrea/vómito 6-12\%). ${ }^{3,4,5}$

El paciente debe mantener un mínimo de 10 días de distanciamiento social previos a su procedimiento electivo.

En caso de cirugía pediátrica, los padres o familiares a cargo del menor también deben ser evaluados según protocolos para detección de virus.

\section{Grupos de riesgo}

Se sabe que las comorbilidades tienen impacto negativo en la resolución en pacientes infectados por COVID-19. Por lo tanto, se deben tomar en cuenta posibles aspectos de riesgo.

\section{Edad}

La mortalidad va de 1.8 a $12.2 \%$ en el grupo etario de 0 a 59 años, aumentando hasta $18.7 \%$ en el grupo etario de 60 a 69 años e incrementa gradualmente con la edad con cifras superiores a $50 \%$ en pacientes mayores de 90 años; este análisis se realizó por década. ${ }^{6}$ Se observa el punto de inflexión a los 65 años, cuando aumentan los casos severos, en $37 \%$ en el grupo etario de 45 a 64 años y en $50.2 \%$ a partir de los 65 años de edad.

\section{Diabetes mellitus}

La diabetes se asocia a mayor riesgo de contracción de enfermedades infecciosas y a mayor severidad y mortalidad en síndrome respiratorio agudo grave (SARS). ${ }^{8}$ Con la información recolectada actualmente sobre COVID-19 se aprecia al paciente diabético como población de riesgo a cuadros de severidad y muerte. En un estudio comparativo de 174 pacientes con y sin diabetes mellitus infectados por COVID-19 se encontró $16.7 \%$ más mortalidad en pacientes con diabetes mellitus. ${ }^{9} \mathrm{Al}$ analizar las cifras laboratoriales proinflamatorias se detectó mayor deterioro en estos pacien-

\begin{tabular}{|c|c|c|c|c|}
\hline Categoría & A & B & $\mathrm{C}$ & $\mathrm{D}$ \\
\hline Urgencia & Emergencia & Urgencia & Urgencia electiva & Electiva \\
\hline $\begin{array}{l}\text { Tipo de } \\
\text { procedimiento }\end{array}$ & $\begin{array}{l}\text { Riesgo de muerte o } \\
\text { pérdida de extremidad }\end{array}$ & $\begin{array}{l}\text { - Trauma } \\
\text { - Infecciones }\end{array}$ & $\begin{array}{l}\text { Rupturas agudas intra/periarticulares } \\
\text { de ligamentos o tendones }\end{array}$ & $\begin{array}{l}\text { Artroplastías } \\
\text { Rupturas crónicas ligamentarias/ } \\
\text { tendinosas } \\
\text { Osteotomías } \\
\text { Síndromes nerviosos compresivos }\end{array}$ \\
\hline
\end{tabular}




\begin{tabular}{|lccccc}
\hline Tabla 2: Riesgo de exposición a COVID-19. & \\
\hline Categoría & 1 & 2 & 3 & 4 \\
\hline Definición & $\begin{array}{c}\text { Sin exposición o } \\
\text { infección previa }\end{array}$ & $\begin{array}{c}\text { Con exposición, } \\
\text { pero asintomático }\end{array}$ & $\begin{array}{c}\text { Recuperado de la infección } \\
\text { y posiblemente inmune }\end{array}$ & $\begin{array}{c}\text { Posiblemente infectado } \\
\text { (test negativo con síntomas) }\end{array}$ & Infectado \\
\hline
\end{tabular}

tes observando mayor progresión a complicaciones con cuadros más severos y de mayor mortalidad. ${ }^{10}$

\section{Hipertensión y enfermedades cardiovasculares}

Si bien es cierto se leen múltiples reportes de mayor severidad y mortalidad en pacientes con hipertensión y enfermedades cardiovasculares, ${ }^{11,12}$ no se ha estudiado la hipertensión como factor aislado de riesgo, tomando en cuenta que es una patología de alta prevalencia en pacientes mayores de 65 años. Uno de los postulados que explica a los hipertensos como grupo de riesgo, es que el virus se une a sus células blanco a través de la enzima convertidora de angiotensina 2 (ECA 2) y un alto porcentaje de pacientes con enfermedad hipertensiva y/o cardiovascular son tratados con bloqueadores del eje renina-angiotensina, ${ }^{13}$ lo cual genera una regulación a la alta de la ECA 2 favoreciendo la vía de infección de COVID-19. Sin embargo, esta teoría no ha sido confirmada. ${ }^{14}$

\section{Índice de masa corporal}

Los pacientes infectados con IMC mayor de 30 presen$\tan$ aumento estadísticamente significativo en su aporte de $\mathrm{O}_{2}$, riesgo a intubación orotraqueal y riesgo de mortalidad en comparación con pacientes con IMC menor de $25 .{ }^{15}$ Asimismo, el riesgo incrementa significativamente para estos tres rubros al comparar paciente de IMC menor de 35 con paciente con IMC mayor de 35. Los pacientes con IMC mayor de 35 tienen 7.36 veces más riesgo de ser intubados que pacientes con IMC menor de 25 y 3.78 veces más riesgo de muerte en comparación con pacientes de IMC de 25 a $34 .{ }^{16}$

\section{Enfermedad pulmonar obstructiva crónica/fumadores}

Una revisión sistemática de 11 artículos publicados con 2002 casos con pacientes con enfermedad pulmonar obstructiva crónica infectados por COVID-19 reveló un riesgo relativo de 4.38 de padecer enfermedad en comparación con pacientes sanos y hasta $60 \%$ de mortalidad en pacientes con enfermedad pulmonar obstructiva crónica. ${ }^{17}$

Tomando en cuenta los estudios publicados, los pacientes fumadores tienen un riesgo relativo de 1.4 de padecer enfermedad severa y 2.4 veces más probable de necesitar intubación orotraqueal, terapia intensiva y/o sufrir muerte. ${ }^{18}$

Cada una de las comorbilidades anteriormente mencionadas representa un riesgo importante para los pacientes, riesgo que aumenta al presentar dos o más comorbilidades. ${ }^{19}$
El cuestionario anexo considera sintomatología de infección activa, categorización de exposición y estratificación de grupo de riesgo. Se recomienda realizar al momento de programación quirúrgica y el día de ingreso hospitalario, en caso de existir datos de riesgo procure diferir el procedimiento (Tabla 3).

Contextualizando esta estratificación de riesgo para nuestro país, la Secretaría de Salud de México reportó al 11 de Junio de 2020, 133,974 casos confirmados (mujeres 44.32\% y hombres $55.68 \%$ ), $20.34 \%$ con hipertensión arterial sistémica, $19.99 \%$ con obesidad definida como IMC mayor de $25,16.80 \%$ con diabetes mellitus y $8.11 \%$ fumadores.

Las 15,944 defunciones $(33.69 \%$ mujeres y $66.31 \%$ hombres) padecían HAS (42.29\%), DM (37.42\%), obesidad $(25.76 \%)$ y tabaquismo $(9.08 \%) .^{20}$

\section{Pruebas de gabinete}

Aparte de la anamnesis dirigida, es de vital importancia agregar estudios de gabinete para descartar infección activa, considerando la existencia de paciente asintomático reportada hasta en $20 \%$ de los casos. ${ }^{21}$

- Pacientes en los que ya descartamos riesgo de exposición o de infección (categoría 1) deberán hacerse la prueba PCR de 48 a 72 horas antes de la cirugía.

- Pacientes que han estado expuestos, pero son asintomáticos (categoría 2) deberán hacerse la prueba PCR y una TAC de tórax de 48 a 72 horas antes de la cirugía.

- Pacientes recuperados de la infección y quizás adecuadamente inmunes (categoría 3) deberán comprobar su inmunidad con prueba serológica de IgG y la negatividad de la prueba PCR antes de la cirugía.

- Pacientes con síntomas y PCR negativa (categoría 4) deben repetir la prueba y una TAC de tórax antes de considerar cualquier cirugía y si se encuentra algún dato pulmonar con relación a COVID-19, se debe diferir la cirugía.

- Por último, las cirugías en pacientes infectados (categoría 5) deben ser retrasadas hasta la total recuperación (por lo menos dos semanas) y después realizar prueba PCR y TAC de tórax de 48 a 72 horas antes de la cirugía. ${ }^{22}$

Se ha observado que algunos pacientes con PCR negativa muestran anormalidades en la TAC de tórax cuestionando la sensibilidad de la PCR, así como reportes de hasta $63 \%$ de falsos negativos. ${ }^{23}$ Los datos tomográficos presentes en pacientes infectados son: opacidad en vidrio despulido en $(86 \%)$ con consolidaciones en $54 \%$ y sin consolidaciones en $39 \%$, engrosamiento vascular ( $82 \%$ ), engrosamiento sep- 
tal interlobular con patrón de pavimentado (70\%) y broncograma aéreo $(68 \%)$ principalmente..$^{24,25}$ La tomografía tiene resultados inmediatos, ya que puede detectar pacientes a última hora. Se reportan falsos negativos en 3.9\% haciendo una prueba confiable y sensible para neumonía viral, pero poco específica para COVID y dependiendo del observador.

Parece que la combinación de PCR y TAC de tórax muestra la mayor sensibilidad diagnóstica (92\%) en comparación con la prueba PCR única $(78 \%)$ o sólo la TAC de tórax $(67 \%)$ o la combinación de dos pruebas PCR (86\%). Es por eso que la combinación de prueba PCR y TAC de tórax es la recomendación para evitar llevar un paciente infectado a cirugía electiva. ${ }^{22}$

\section{Recomendaciones intraoperatorias}

En cuanto a las características de los quirófanos el sistema de ventilación con presión positiva para cirugía electiva es efectivo, no es necesario realizar la conversión a quirófanos con presión negativa para estos pacientes.

Se recomienda disminuir el personal al esencial dentro del quirófano, especialmente durante la intubación y extubación del paciente, así como usar una sola puerta para evitar contaminación en pasillos y corredores.

El uso de equipo de protección personal en áreas de alta prevalencia de contagio por COVID-19, los cirujanos y todo el equipo quirúrgico deben protegerse con mascarillas N95, FFP2 o FFP3, caretas, gafas y usar protección para cuello y zonas expuestas a salpicaduras o contaminación con fluidos. Conforme vaya disminuyendo el riesgo de contagio de acuerdo a cada zona del país y en pacientes con prueba PCR negativa, TAC no compatible con COVID-19 y con distanciamiento social de 10 días previo a cirugía, podrá realizarse la cirugía con el equipo de protección estándar. La evidencia actual no recomienda el uso de escafandras o cascos con ventilación, debido a la dificultad para su esterilización y a la ausencia de filtros y/o métodos de purificación de los mismos para evitar el contagio al cirujano. ${ }^{26}$

\section{Anestesia}

El objetivo principal para la selección de la técnica anestésica es mantener la seguridad del paciente y del personal de salud. Dado que el COVID-19 se transmite vía aerosol con contacto cercano de una persona a otra, es preferible evitar la instrumentación de la vía aérea. Por lo tanto, cuan-

\begin{tabular}{|c|c|c|c|c|c|}
\hline \multicolumn{6}{|c|}{ Tabla 3: Riesgo por comorbilidad. } \\
\hline Factor & Edad & DM & EPOC & IMC & HTA \\
\hline Corte & $>65$ años & & & $>30$ & \\
\hline Riesgo & ++++ & ++++ & ++++ & ++++ & ++ \\
\hline
\end{tabular}

do sea posible se debe optar por anestesia regional, ya sea bloqueo de nervios periféricos o bloqueos neuroaxiales.

El paciente debe usar cubrebocas durante el procedimiento. Se debe evitar el uso de oxígeno suplementario, si es necesario se colocará por medio de puntas nasales por debajo del cubrebocas y se debe administrar el flujo más bajo sólo para mantener la saturación de oxígeno. Se ha demostrado que la distancia alcanzada por aire exhalado aumenta con el incremento del flujo de oxígeno, llegando hasta 0.4 metros con flujos de $10 \mathrm{~L} / \mathrm{min}^{27}$

El anestesiólogo debe al menos colocarse cubrebocas y gafas. El uso de respirador N95 será a discreción del operador, ya que la anestesia regional no es un procedimiento generador de aerosoles. El uso de caretas o cascos puede asociarse con reducción de visibilidad o audición, ocasionando mala comunicación y poca movilidad del anestesiólogo. La combinación de estos factores puede afectar la concentración y la destreza del operador. ${ }^{28}$

Previo el inicio de la cirugía se debe probar la eficacia del bloqueo, tratando de evitar conversión a anestesia general (AG) de urgencia cuando la cirugía ya inició. En caso de requerir la conversión a AG el anestesiólogo deberá colocarse el EPP recomendado por la Sociedad Americana de Anestesiología, que incluye respirador N95, protección ocular con gafas o careta, bata y guantes. Se debe realizar higiene de manos antes y después de colocarse el EPP. ${ }^{29}$

Al elegir AG como técnica anestésica se recomienda evitar las situaciones que produzcan aerosoles como la ventilación con bolsa o el uso de supraglóticos. La técnica adecuada es la intubación seguida de inducción de secuencia rápida, así evitamos cualquier fuga en el circuito. Se recomienda utilizar filtros HEPA (por sus siglas en inglés High Efficiency Particulate Air) en el extremo del gas exhalado para disminuir aún más el riesgo de aerosolización. Se debe considerar el uso de videolaringoscopio, si está disponible, para disminuir el tiempo de intubación. ${ }^{30}$

La elección de técnica de mantenimiento (anestesia inhalada o anestesia total intravenosa) depende del escenario clínico. Sin embargo, el objetivo principal debe ser una rápida emersión evitando que el paciente tosa.

Como profilaxis antiemética, se recomienda la utilización de dos o tres fármacos porque el vómito postoperatorio puede generar aerosoles. Asimismo, se debe considerar evitar el uso de opioides para el control de dolor postoperatorio, ya que pueden causar depresión respiratoria que requiera manipulación de la vía aérea. ${ }^{27}$

\section{Acto quirúrgico}

El uso del electrocoagulador genera aerosoles, aunque no se ha demostrado que aumente el riesgo de infección intraoperatoria, se recomienda minimizar su uso y en caso de ser necesario se debe usar con un sistema adecuado de aspiración de humo. Los instrumentos de alta potencia son igualmente posibles generadores de aerosoles; la recomendación es considerar el uso de sierras manuales (Gigli, osteótomos, 
fresados y rimados manuales) o emplear los motores a baja velocidad y frecuencia. Disminuya o evite el uso de sutura no absorbible o grapas con el fin de evitar visitas postquirúrgicas al hospital y/o consultorios. ${ }^{26,31}$

El instrumental quirúrgico requiere esterilización estándar, procurando una descontaminación en áreas determinadas para su procesamiento después de su uso con el fin de evitar que sangre o fluidos corporales puedan contaminar al personal o superficies. Toda solución para descontaminación postquirúrgica del instrumental se debe desechar por rutas y en sitios adecuados. ${ }^{32}$

Realizar limpieza exhaustiva de equipos de rayos $\mathrm{X}$, trajes plomados, mesas de transporte y equipo de proveedores. El equipo de rayos $\mathrm{X}$ debe permanecer siempre dentro de quirófano y se debe asear completamente a la brevedad posible una vez terminada la cirugía. ${ }^{31}$

\section{Postoperatorio}

En su traslado al área de recuperación se recomienda que el paciente use cubrebocas. Se debe minimizar la estadía en zonas de recuperación postoperatoria procurando el pase directo a la habitación, si es posible. Favorezca e incremente zonas de recuperación no COVID-19 y cirugía ambulatoria para minimizar la estancia hospitalaria. Limite visitas de familiares o el tiempo de las mismas dentro del hospital, procurando la vía telefónica o videollamada para la comunicación con familiares y así minimizar el contacto y/o exposición innecesaria. En cuanto al manejo farmacológico postoperatorio no existen restricciones actuales específicas para manejo antiinflamatorio, profilaxis antibiótica o tromboprofilaxis ${ }^{31}$ por lo que se recomienda seguir con los protocolos establecidos de manera habitual.

El pase de visita debe ser expedido de preferencia por un solo médico, procurando reforzar la educación de medidas de higiene a familiares y cuidadores para disminuir riesgo de exposición y contagio.

Establezca comunicación y educación a terapeutas físicos, personal de enfermería o auxiliares en el cuidado en casa para una rehabilitación adecuada del paciente.

\section{Conclusiones}

Establecer guías y algoritmos adecuados nos ayuda a disminuir el riesgo de contagios de todo el personal involucrado en el desarrollo de la cirugía del paciente así como aprovechar y evitar el uso innecesario del equipo de protección personal ocasionando desabasto.

Todos los algoritmos o guías tienen probabilidad de falla porque en ocasiones es imposible determinar el estado infeccioso de un paciente, tomando en cuenta que las pruebas tienen un porcentaje de falsos negativos (hasta 63\%) y considerando que $20 \%$ los pacientes infectados son asintomáticos, por lo que lo primordial es tratar de ajustar estas recomendaciones de acuerdo a las pruebas de cada hospital, institución y situación local.
Se trata de un padecimiento nuevo para la humanidad y por lo tanto, el tratamiento de la patología está en constante cambio, representando un gran reto adaptarse y establecer un protocolo o algoritmo real de tratamiento a seguir, por lo tanto, debemos permanecer informados y apegados a las recomendaciones comprobadas.

Al tratarse de cirugía electiva debemos entender que contamos con el tiempo suficiente para analizar el caso y seguir las recomendaciones al pie de la letra. El trabajo preoperatorio de análisis profundo del caso, considerando el tipo de cirugía, las instalaciones institucionales y el riesgo de infección del paciente, desempeña un papel esencial para minimizar el riesgo del personal de salud. El paciente programado para cirugía electiva debe permanecer en distanciamiento social mínimo de 10 días considerando el período de ventana de la patología y así lograr minimizar el riesgo de contagio.

En pacientes con presencia de síntomas o criterios mayores (fiebre, tos seca o dolor de garganta u odinofagia, prueba PCR positiva o TAC sospechosa) se debe retrasar la cirugía hasta la resolución del cuadro infeccioso.

Actualmente en un período de 24 a 36 horas podemos obtener los resultados de las pruebas PCR, por lo que en todos los casos de cirugía electiva podemos esperar los resultados de las pruebas, recalcando que no debemos exponer innecesariamente al paciente ni al personal de salud.

Bibliografía

1. AAOS Guidelines for Elective Surgery-American Academy of Orthopaedic Surgeons. [Accessed 30 Apr 2020] Available in: https:// www.aaos.org/about/covid-19information-for-our-members/aaosguidelines-for-elective-surgery/.

2. Fineberg HV. Ten weeks to crush the curve. $N$ Engl J Med. 2020; 382: e37.

3. Rodriguez-Morales AJ, Cardona-Ospina JA, Gutiérrez-Ocampo E, et al. Clinical, laboratory and imaging features of COVID-19: a systematic review and meta-analysis. Travel Med Infect Dis. 2020; 34: 101623. doi: 10.1016/j.tmaid.2020.101623.

4. Guan WJ, Liang WH, Zhao Y, et al. Comorbidity and its impact on 1590 patients with COVID-19 in China: a nationwide analysis. Eur Respir J. 2020; 55(5): 2000547. Published 2020 May 14. doi: 10.1183/13993003.00547-2020.

5. Yang J, Zheng Y, Gou X, et al. Prevalence of comorbidities and its effects in patients infected with SARS-CoV-2: a systematic review and meta-analysis. Int J Infect Dis. 2020; 94: 91-95. doi: 10.1016/j. ijid.2020.03.017.

6. Richardson S, Hirsch JS, Narasimhan M, et al. Presenting Characteristics, Comorbidities, and Outcomes Among 5700 Patients Hospitalized With COVID-19 in the New York City Area [published online ahead of print, 2020 Apr 22]. JAMA. 2020; e206775. doi: 10.1001/jama.2020.6775.

7. Li X, Xu S, Yu M, et al. Risk factors for severity and mortality in adult COVID-19 inpatients in Wuhan [published online ahead of print, 2020 Apr 12]. J Allergy Clin Immunol. 2020; S0091-6749(20)30495-4. doi: 10.1016/j.jaci.2020.04.006.

8. Muller LM, Gorter KJ, Hak E, et al. Increased risk of common infections in patients with type 1 and type 2 diabetes mellitus. Clin Infect Dis. 2005; 41(3): 281-288.

9. Yang JK, Feng Y, Yuan MY, et al. Plasma glucose levels and diabetes are independent predictors for mortality and morbidity in patients with SARS. Diabetic Med. 2006; 23(6): 623-628.

10. Guo W, Li M, Dong Y, et al. Diabetes is a risk factor for the progression and prognosis of COVID-19. [published online ahead 
of print, 2020 Mar 31]. Diabetes Metab Res Rev. 2020; e3319. doi: 10.1002/dmrr.3319.

11. Zhou F, Yu T, Du R, Fan G, Liu Y, Liu Z, et al. Clinical features of patients infected with 2019 novel coronavirus in Wuhan, China. Lancet. 2020; 395: 497-506.

12. Wu C, Chen X, Cai Y, Xia J, Zhou X, Xu S, et al. Risk factors associated with acute respiratory distress syndrome and death in patients with coronavirus disease 2019 pneumonia in Wuhan, China. JAMA Intern Med. 2020, published online 13 March. doi: 10.1001/ jamainternmed.2020.0994.

13. Fang L, Karakiulakis G, Roth M. Are patients with hypertension and diabetes mellitus at increased risk for COVID-19 infection? [published correction appears in Lancet Respir Med. 2020;. Lancet Respir Med. 2020; 8(4): e21. doi: 10.1016/S2213-2600(20)30116-8.

14. Schiffrin EL, Flack JM, Ito S, Muntner P, Webb RC. Hypertension and COVID-19. Am J Hypertens. 2020; 33(5): 373-374. doi: 10.1093/ajh/ hpaa057.

15. Simonnet A, Chetboun M, Poissy J, et al. High prevalence of obesity in severe acute respiratory syndrome coronavirus-2 (SARS-CoV-2) requiring invasive mechanical ventilation. [published online ahead of print, 2020 Apr 9]. Obesity (Silver Spring). 2020; 10.1002/oby.22831.

16. Palaiodimos L, Kokkinidis DG, Li W, et al. Severe obesity, increasing age and male sex are independently associated with worse in-hospital outcomes, and higher in-hospital mortality, in a cohort of patients with COVID-19 in the Bronx, New York [published online ahead of print, 2020 May 16]. Metabolism. 2020; 108: 154262. doi: 10.1016/j.metabol.2020.154262.

17. Zhao Q, Meng M, Kumar R, et al. The impact of COPD and smoking history on the severity of COVID-19: a systemic review and metaanalysis [published online ahead of print, 2020 Apr 15]. J Med Virol. 2020; 10.1002/jmv.25889. doi: 10.1002/jmv.25889.

18. Vardavas CI, Nikitara K. COVID-19 and smoking: a systematic review of the evidence. Tab Induc Dis. 2020; 18: 20. Published 2020 Mar 20. doi: 10.18332/tid/119324.

19. Guan WJ, Liang WH, Zhao Y, et al. Comorbidity and its impact on 1590 patients with COVID-19 in China: a nationwide analysis. Eur Respir J. 2020; 55(5): 2000547. Published 2020 May 14. doi: $10.1183 / 13993003.00547-2020$.

20. Gobierno de México. (2020, May 21). COVID-19 México. Retrieved June 11, 2020, from https://coronavirus.gob.mx/datos/.

21. Kim GU, Kim MJ, Ra SH, et al. Clinical characteristics of asymptomatic and symptomatic patients with mild COVID-19 [published online ahead of print, 2020 May 1]. Clin Microbiol Infect. 2020; S1198-743X(20)30268-8. doi: 10.1016/j.cmi.2020.04.040.

22. Mouton C, Hirschmann MT, Ollivier M, Seil R, Menetrey J. COVID19-ESSKA guidelines and recommendations for resuming elective surgery. J Exp Orthop. 2020; 7(1): 28. Published 2020 May 13. doi: 10.1186/s40634-020-00248-4.

23. Li Y, Yao L, Li J, et al. Stability issues of RT-PCR testing of SARSCoV-2 for hospitalized patients clinically diagnosed with COVID-19. J Med Virol. 2020; 92(7): 903-908. doi: 10.1002/jmv.25786.

24. Kanne JP. Chest CT Findings in 2019 Novel coronavirus (2019$\mathrm{nCoV}$ ) infections from Wuhan, China: key points for the radiologist. Radiology. 2020; 295(1): 16-17. doi: 10.1148/radiol.2020200241.

25. Li Y, Xia L. Coronavirus Disease 2019 (COVID-19): role of chest CT in diagnosis and Management. AJR Am J Roentgenol. 2020; 214(6): 1280-1286. doi:10.2214/AJR.20.22954.
26. Coimbra R, Edwards S, Kurihara H, Bass GA, Balogh ZJ, Tilsed J, et al. European Society of Trauma and Emergency Surgery (ESTES) recommendations for trauma and emergency surgery preparation during times of COVID-19 infection. Eur J Trauma Emerg Surg. 2020; [Epub ahead of print]

27. Rajan N, Joshi GP. The COVID-19: role of ambulatory surgery facilities in this global pandemic. [published online ahead of print, 2020 Apr 1]. Anesth Analg. 2020; 10.1213/ANE.0000000000004847. doi: 10.1213/ANE.0000000000004847.

28. Lie SA, Wong SW, Wong LT, Wong TGL, Chong SY. Practical considerations for performing regional anesthesia: lessons learned from the COVID-19 pandemic. Considérations pratiques pour laréalisation de l'anesthésie régionale: les leçons tirées de la pandémie de COVID-19. Can J Anaesth. 2020; 67(7): 885-892. doi: 10.1007/ s12630-020-01637-0.

29. ASA UPDATE: The use of personal Protective Equipmentby Anesthesia Professionals during the COVIDPandemic. [Accessed March 29, 2020] Available at: https://www.asahq.org/about-asa/ newsroom/news-releases/2020/03/updatethe-use-of-personalprotective-equipment-by-anesthesiaprofessionalsduring-the-covid-19 pandemic? \&ct=898dd13e2a0ac20c595b3180cd23a166d7369e568b 7eb5ea2bf3b5f5e1ed3c34f0b0fdd5c2769b94df300888aed43c14c890 84d0e016ccf0494f3c757926550b.

30. Edelson DP, Sasson C, Chan PS, et al. Interim Guidance for Basic and Advanced Life Support in Adults, Children, and Neonates With Suspected or Confirmed COVID-19: From the Emergency Cardiovascular Care Committee and Get With the Guidelines ${ }^{\circledR}$ Resuscitation Adult and Pediatric Task Forces of the American Heart Association in Collaboration with the American Academy of Pediatrics, American Association for Respiratory Care, American College of Emergency Physicians, The Society of Critical Care Anesthesiologists, and American Society of Anesthesiologists: Supporting Organizations: American Association of Critical Care Nurses and National EMS Physicians [published online ahead of print, 2020 Apr 9]. Circulation. 2020; 10.1161/CIRCULATIONAHA.120.047463. doi: 10.1161/ CIRCULATIONAHA.120.047463.

31. Parvizi J, Gehrke T, Krueger CA, et al. Resuming Elective Orthopaedic Surgery During the COVID-19 Pandemic: Guidelines Developed by the International Consensus Group (ICM) [published online ahead of print, 2020 May 14]. J Bone Joint Surg Am. 2020; 10.2106/JBJS.20.00844. doi: 10.2106/ JBJS.20.00844.

32. From the American Association of Neurological Surgeons (AANS), American Society of Neuroradiology (ASNR), Cardiovascular and Interventional Radiology Society of Europe (CIRSE), Canadian Interventional Radiology Association (CIRA), Congress of Neurological Surgeons (CNS), European Society of Minimally Invasive Neurological Therapy (ESMINT), European Society of Neuroradiology (ESNR), European Stroke Organization (ESO), Society for Cardiovascular Angiography and Interventions (SCAI), Society of Interventional Radiology (SIR), Society of NeuroInterventional Surgery (SNIS), and World Stroke Organization (WSO), Sacks D, Baxter B, et al. Multisociety Consensus Quality Improvement Revised Consensus Statement for Endovascular Therapy of Acute IschemicStroke. Int J Stroke. 2018; 13(6): 612-632. doi: $10.1177 / 1747493018778713$. 
Día de programación de cirugía

$24 \mathrm{~h}$ previas a procedimiento

Factores de riesgo

DM

HAS

Obesidad

$>65$ años

EPOC

Fumador

Inmunosupresión

Sin exposición previa

Expuesto asintomático

Recuperado de infección

Posiblemente infectado

Infectado

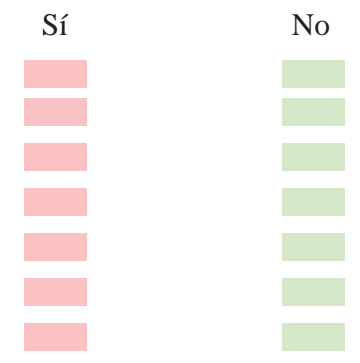

\section{Riesgo de exposición}

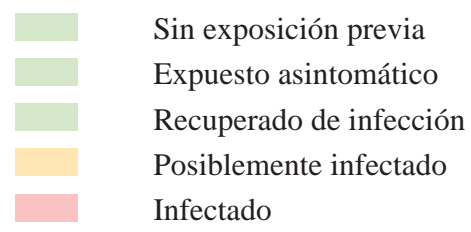

Signos y síntomas

Fiebre

Tos

Fatiga

Disnea

Mialgias/artralgias

Cefalea

Hiposmia/hipogeusia

Sx gastrointestinales

\begin{tabular}{|l|l} 
Sí & No \\
\hline & Fiebre \\
& Tos \\
& Fatiga \\
& Disnea \\
& Mialgias/artralgias \\
& Cefalea \\
\hline & Hiposmia/hipogeusia \\
\hline & Sx gastrointestinales \\
\hline &
\end{tabular}

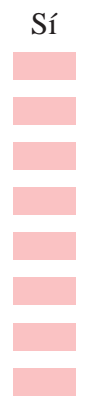

No

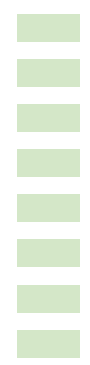

Positiva

Negativa

PCR

TAC

DM = diabetes mellitus, HAS = hipertensión arterial sistémica, EPOC = enfermedad pulmonar obstructiva crónica, PCR = reacción en cadena de la polimerasa, TAC = tomografía axial computarizada. 